\title{
Construcción de la Escala de Adicción al Trabajo en adultos
}

\author{
Construction of the Work Addiction Scale in adults
}

\section{Brandon Apaza Ramirez¹, Maryuri Pérez Coaquira ${ }^{2}$}

\begin{abstract}
RESUMEN
Objetivo: Construir y evaluar de las propiedades psicométricas de un instrumento que evalúa la adicción al trabajo en jóvenes y adultos entre las edades de 20 a 66 años, que sean necesariamente trabajadores. Metodología: En la primera etapa del estudio se realizó la delimitación conceptual del constructo y la elaboración preliminar instrumento (8 ítems) con cuatro categorías de respuesta. En la segunda etapa, se administró a una muestra piloto de 239 personas. Resultados: La validez de contenido, obtuvo puntajes por encima de (.80), asimismo, se efectuó el análisis factorial exploratorio a fin de someter a un análisis riguroso la naturaleza teórica y dimensional del constructo, alcanzando en la Medida de adecuación muestral de KMO un puntaje de (.891), también se aprecia que los ítems forman un solo factor que explica el $45.7 \%$ de la varianza y donde sus pesos factoriales son elevados ( >.50). Por último, se obtuvo el coeficiente alfa de Cronbach (.868), siendo un puntaje valorado como indicador de una elevada fiabilidad. Conclusión: El Test de Adicción al Trabajo evidencia buena consistencia interna y validez de constructo.
\end{abstract}

Palabras clave: Adicción, trabajo, obsesión, compulsión, ansiedad, aislamiento.

\begin{abstract}
Objective: to construct and evaluate the psychometric properties of an instrument that evaluates work addiction in young people and adults between the ages of 20 to 66 , who are necessarily workers. Methodology: in the first stage of the study, the conceptual delimitation of the construct and the preliminary preparation of the instrument ( 8 items) with four response categories were carried out. In the second stage, a pilot sample of 239 people was administered. Results: content validity, obtained scores above ( .80$)$, also, exploratory factor analysis was carried out in order to submit the theoretical and dimensional nature of the construct to a rigorous analysis, reaching the KMO Sample Adequacy Measure a score of (.891), it is also seen that the items form a single factor that explains $45.7 \%$ of the variance and where their factor weights are high (> .50). Finally, the Cronbach's alpha coefficient (.868) was obtained, being a valued score as an indicator of high reliability. Conclusion: the Work Addiction Test shows good internal consistency and construct validity.
\end{abstract}

Keywords: Addiction, work, obsession, compulsion, anxiety, isolation.

${ }^{1}$ Universidad Peruana Unión, Lima, Perú.

Orcid ID: 0000-0002-6528-0002

${ }^{2}$ Universidad Peruana Unión, Lima, Perú.

Orcid ID: 0000-0003-0942-7003 


\section{INTRODUCCIÓN}

La adicción al trabajo se manifiesta en distintas culturas, para tener una idea, la cantidad de ejecutivos adictos al trabajo en Estados Unidos es $23.0 \%$ de su población económicamente activa (PEA), de acuerdo con Doerfler y Kramer (1986); por otro lado, Kanai, Wakabayashi y Fling (1996) reportaron, en una muestra de más de 600 participantes en Japón compuesta principalmente por gerentes, que la cifra de adictos al trabajo fue de $21 \%$. En México, tomando el criterio propuesto por Mosier (1983), que considera como adicto al trabajo a la persona que trabaja más de 48 horas por semana, se estima que $28 \%$ de la PEA del país entra en el grupo de adictos al trabajo (INEGI, 2012), que es una proporción significativa de los trabajadores mexicanos.

Por otro lado, Castillo y Gómez (2012) encontraron en su estudio sobre adicción al trabajo diferencias entre dos grupos, el de puestos ejecutivos/ administrativos (EA) y el de puestos operativos (O). Siendo los miembros del grupo EA un $67 \%$ encima de la media, mientras que sólo el 33\% de los miembros del grupo $O$ superan la media. Por lo tanto, estos porcentajes muestran que la adicción al trabajo, son más evidentes en la población que pertenece al grupo de puestos de trabajo administrativo / ejecutivos.

Este problema presenta consecuencias en diversos niveles, en lo fisiológico, según Scott, Moore y Miceli (1997) cansancio crónico, estrés, trastornos sexuales, insomnio y trastornos psicosomáticos. En el nivel cognitivo-emocional, Del Líbano, Llorens, Schaufeli y Salanova (2006), indican que presentan ansiedad, miedo al fracaso, baja autoestima, irritabilidad, intolerancia, disminución de la memoria y depresión; asimismo, a nivel comportamental se refleja disminución en el rendimiento laboral, ya que el individuo se siente cansado ya sea físicamente o emocionalmente, también desarrolla otras conductas adictivas, al sexo, consumo de alcohol, fármacos y otras drogas. Además, se manifiesta con otros tipos de conductas como necesidad de realizar varias tareas simultáneamente, delegar trabajos, dificultad para relajarse, incapacidad para solucionar problemas, desinterés en las relaciones interpersonales, deterioro en el núcleo familiar y aislamiento social. Del mismo modo, Fassel (1990) la considera como una enfermedad fatal que aleja a las personas de sus familiares y amigos.

Frente a esta problemática se desarrollaron diversas pruebas que pretenden medir esta variable, en primer lugar tenemos la de Robinson, que desarrolló el The Work Addiction Risk Test (WART) para medir las tendencias de adicción al trabajo y los patrones de trabajo adictivos. Los productos del WART se recopilaron a partir de una lista de síntomas reportados por los profesionales que trabajan con las familias afectadas por la adicción al trabajo. EI WART es una medida válida y fiable de la adicción al trabajo. La puntuación para el WART se obtiene normalmente mediante la suma de las respuestas para cada uno de los 25 elementos para calcular una puntuación global (Aziz, Adkins, Walker, y Wuensch, 2010).

Los estudios sobre el WART han encontrado que constan de dimensiones subyacentes que resultan en cinco subescalas: tendencias compulsivas, incapacidad para controlar los hábitos de trabajo, problemas de comunicación, incapacidad para delegar, y deterioro de la autoestima (Flowers y Robinson, 2002)

Por otro lado, tenemos el cuestionario Duwas-10 (Dutch Work Addiction Brief Scale of 10 item) instrumento que se seleccionó para medir la adicción al trabajo, propuesto y validado por Del Líbano, Llorens, Salanova y Schaufeli, que se evalúa con una escala de Likert de 1 a 4 , en la que 1 es "totalmente en desacuerdo" y 4 es "totalmente de acuerdo", y arroja como resultado dos factores: trabajo en exceso y trabajo compulsivo -éste cuenta con un alfa de Cronbach de 0.75 en su aplicación en Holanda y 0.85 en España (citado por Colín y Simón, 2014)

Por lo expuesto, se evidencia que la adicción al trabajo afecta a un porcentaje considerable de trabajadores en diversas culturas, además que genera consecuencias negativas a nivel fisiológico, cognitivo y comportamental, lo que deteriora el núcleo familiar y conlleva a un aislamiento social.

Es por eso que este estudio pretende brindar una herramienta capaz de identificar la adicción al trabajo en nuestra cultura peruana. $Y$ esto puede servir como una base para crear talleres o actividades que mejoren el estilo de vida en los trabajadores peruanos. 


\section{Conceptualización de la variable}

La adicción al trabajo, según Oates (1971), es una necesidad excesiva e incontrolable de trabajar incesantemente, Sorensen, y Feldman (2006) señalan que hay una obsesión por el trabajo, y Fassel (citado por Merino, Boada \& Prizmic, 2014) lo define como una patología. Por ende, conlleva a realizar actos y pensamientos compulsivos (Quinceno y Vinaccia, 2007).

Para Schaufeli, Bakker, van der Heijden y Prins (2009) la peculiaridad más obvia en estas personas es que trabajan más de lo que se requiere, gastando una cantidad excesiva de tiempo y energía. El adicto al trabajo no está motivado por factores externos (problemas financieros o en el matrimonio, presión social, promoción profesional), si no por una unidad interna obsesiva que no se puede resistir. Por otro lado, una de las aportaciones más significativas fue el modelo que contempla tres dimensiones importantes, realizada por Spence y Robbins (1992) que son: Implicación en el trabajo (work involvement), Disfrute con el trabajo (work enjoyment) e Impulso (drive).

Por lo tanto, se puede decir que es una enfermedad caracterizada por una obsesión persistente e incontrolable hacia el trabajo, que conlleva al individuo a realizar actos compulsivos. Generando estrés, ansiedad y cansancio crónico; así mismo deteriora el núcleo familiar y conlleva a un aislamiento social.

\section{METODOLOGÍA}

La presente investigación tiene un enfoque cuantitativo y un diseño no experimental ya que no se manipula la variable, además es de tipo Psicométrico y de corte transversal, puesto que la recolección de datos se realizará en un momento único (Hernández, Fernández, y Baptista, 2006).

\section{Participantes}

El estudio piloto se realizó a trabajadores de diversas áreas de una universidad privada de Lima Este. Para la selección de los participantes se empleó el método no probabilístico por conveniencia, de esta manera se contó con un total de 239 participantes adultos de ambos sexos, desde la adultez temprana hasta la tardía, desde una edad mínima de 20 años a una edad máxima de 66; en cuanto al sexo, la muestra está conformada por 146 varones $(61 \%)$ y 93 mujeres (39\%).

\section{Proceso de construcción}

Para obtener la validez de contenido, se recurrió al criterio de expertos. El grupo de jueces estuvo conformado por: cinco psicólogos, dos del área de investigación, dos del área organizacional y uno del área clínica.

Luego se procedió a realizar el estudio piloto, en el que se obtuvo una muestra de 239 trabajadores. Este procedimiento se inició solicitando un permiso de parte de la Directora de Investigación de la Escuela de Psicología de dicha Universidad, para tener la autorización de poder aplicar el test. El permiso fue aceptado después de 3 días y luego se procedió a encuestar a los trabajadores en sus horarios disponibles.

Después de recabar la información, se procedió a analizar los datos. Así pues, para el análisis de fiabilidad se usó el Alpha de Cronbach, para la validez de contenido se utilizó el método de validez $V$ de Aiken, para la validez de constructo se realizó un análisis factorial exploratorio, usando el método de mínimos cuadrados no ponderados, con rotación oblimín, y para determinar la cantidad de factores se utilizó el análisis de correspondencia, para evaluar la estructura interna de la escala, para lo cual se corroboró el cumplimiento de los supuestos necesario para realizar este análisis, la prueba KMO y la prueba de esfericidad de Bartlet. Todos estos análisis se realizaron mediante el programa estadístico SPSS.

\section{RESULTADOS}

\section{Validez de contenido}

La validez de contenido se obtuvo mediante el criterio de expertos. El grupo de jueces estuvo conformado por: cinco psicólogos, dos del área de investigación, dos del área organizacional y uno del área clínica.

Escurra (1998) menciona que los reactivos cuyos valores sean mayores o iguales a 0.80 se consideran válidos para el test. De esta 
manera se procedió a calcular los coeficientes mediante el método de validez $\mathrm{V}$ de Aiken para el test, siendo esta unidimensional. Los ítems no presentan coeficientes por debajo de 0.80 , lo cual indica que no presenta dificultad en el enunciado de los ítems, teniendo relación con el constructo, sus palabras son usuales para nuestro contexto y evalúa específicamente el test.
En la tabla 1, vemos el contenido de validez $\mathrm{V}$ para el análisis de contenido respecto al Test de Adicción al Trabajo presenta valores mayores de 0.80 , indicando el consenso que existe relación con el constructo, las palabras son usuales para nuestro contexto y evalúa específicamente el test, obteniendo la validez del contenido del instrumento

Tabla 1

Análisis de contenido del instrumento

\begin{tabular}{ll} 
Test & V \\
Forma correcta de aplicación y estructura & 0.8 \\
Orden de las preguntas establecido adecuadamente & 1 \\
Contiene el test preguntas difíciles de entender & 1 \\
Contiene el test palabras difíciles de entender & 1 \\
Las opciones de respuestas son pertinentes y están suficientemente graduados & 1 \\
Forma correcta de aplicación y estructura & 1 \\
& Jueces: 5 \\
\hline
\end{tabular}

\section{Análisis descriptivo de los Ítems}

En las Tabla 2, se presentan las medias, desviaciones estándar, asimetrías, curtosis y correlación ítem-test corregido de los ítems. El puntaje promedio de los ítems varió entre $1.92(\mathrm{DS}=.860)$ y 2.49 (DS = 1.056). La asimetría y curtosis presentan valores inferiores a + - 1.5 (Pérez \& Medrano, 2010).

Tabla 2

Análisis descriptivo de los ítems

\begin{tabular}{|c|c|c|c|c|}
\hline \multirow{2}{*}{ Ítems } & \multicolumn{4}{|c|}{ Espectador } \\
\hline & $\mathbf{M}$ & DS & As & Cur \\
\hline $\begin{array}{l}\text { 1. Cuando estoy en otras actividades siento la necesidad de volver a } \\
\text { trabajar. }\end{array}$ & 2.49 & 1.004 & 0.066 & -1.061 \\
\hline 2. Pienso constantemente en mi trabajo. & 2.34 & 0.860 & 0.276 & -0.515 \\
\hline 3. Siento preocupación y ganas de trabajar exhaustivamente. & 2.02 & 0.989 & 0.588 & -0.747 \\
\hline 4. Las personas me han dicho que trabajo más de lo que debería. & 2.33 & 0.899 & 0.112 & -0.779 \\
\hline 5. Trabajo más de 10 horas al día, por mi propia cuenta. & 1.92 & 1.013 & 0.731 & -0.700 \\
\hline 6. Me pongo a trabajar, aun cuando no necesito hacerlo. & 2.09 & 1.048 & 0.553 & -0.912 \\
\hline 7. Me quedo trabajando hasta tarde. & 1.94 & 1.056 & 0.744 & -0.758 \\
\hline 8. Prefiero trabajar que hacer vida social. & 2.39 & 0.994 & 0.208 & -0.986 \\
\hline
\end{tabular}

\section{Validez de Constructo}

Se realizó un análisis factorial exploratorio, usando el método de mínimos cuadrados no ponderados, con rotación oblimín, y para determinar la cantidad de factores se utilizó el análisis de correspondencia, para evaluar la estructura interna de la escala, para lo cual se corroboró el cumplimiento de los supuestos necesario para realizar este análisis. Como se aprecia en la Tabla 4, el coeficiente de KMO (.891) evidencia q existe una relación suficientemente elevada entre los ítems. También se aprecia que en la prueba de esfericidad de Bartlett (.001) la matriz analizada no es una matriz de identidad. 
En la Tabla 3 se aprecia que todos los ítems forman un solo factor que explica el $45.7 \%$ de la varianza y donde sus pesos factoriales son elevados (mayor a .50).

Tabla 3

Análisis Factorial

\begin{tabular}{|c|c|}
\hline Ítems & Factor I \\
\hline 1. Cuando estoy en otras actividades siento la necesidad de volver a trabajar. & 0.598 \\
\hline 2. Pienso constantemente en mi trabajo. & 0.543 \\
\hline 3. Siento preocupación y ganas de trabajar exhaustivamente. & 0.663 \\
\hline 4. Las personas me han dicho que trabajo más de lo que debería. & 0.688 \\
\hline 5. Trabajo más de 10 horas al día, por mi propia cuenta. & 0.792 \\
\hline 6. Me pongo a trabajar, aun cuando no necesito hacerlo. & 0.737 \\
\hline 7. Me quedo trabajando hasta tarde. & 0.774 \\
\hline 8. Prefiero trabajar que hacer vida social. & 0.601 \\
\hline$\%$ Total de varianza explicada & $45.7 \%$ \\
\hline Prueba KMO & .891 \\
\hline Prueba de Bartlett & .001 \\
\hline
\end{tabular}

\section{Análisis de fiabilidad}

Para obtener la fiabilidad global de la escala se valoró calculando el índice de consistencia interna mediante el coeficiente Alpha de Cronbach. Los resultados del test de Adicción al Trabajo (8 ítems) en la muestra estudiada, es de .868 , que puede ser valorado como indicador de una elevada fiabilidad; asimismo

\section{DISCUSIÓN}

La adicción al trabajo es una problemática que está siendo cada vez más atendida y estudiada por especialistas de la salud ocupacional (Salas y Copez, 2018), en el Perú, no hay investigaciones que mencionen la incidencia o prevalencia de dicha variable, sin embargo, la Dirección de Investigación Socio Económica Laboral brinda un porcentaje de $36 \%$ de la PEA que sobrepasa las 48 horas semanales de trabajo (Ministerio del Trabajo, 2016), y que encaja en la definición de adicción al trabajo de Mosier (1983). Con la construcción psicométrica de esta escala, se pretende iniciar investigaciones que amplíen el conocimiento de dicha problemática.

Los resultados de la construcción psicométrica de la Escala de Adicción al Trabajo fueron favorables, presentando valores por encima de
.80 en la Validez de Contenido (Escurra,1998), asimismo, al realizar el análisis paralelo, se encontró que los ítems se ordenan en un solo factor, por lo que se consideró a la escala como unidimensional (Merino y Domínguez, 2015), del mismo modo, los pesos factoriales de los ítems son mayores de .50 en el análisis factorial exploratorio y se obtuvo una fiabilidad elevada con un puntaje de .868 .

La escala es una herramienta eficaz para detectar una obsesión persistente e incontrolable hacia el trabajo, con conductas compulsivas que producen aislamiento social. (Sorensen y Feldman, 2006; Quinceno y Vinaccia, 2007; Schaufeli y Salanova, 2006).

Se sugiere que en futuras investigaciones se amplíe la muestra, ya que en este caso fue relativamente baja, con una muestra de mayor tamaño se podrían generalizar los resultados en una población. Como una limitación, la escala no cuenta con dimensiones, por lo que se recomienda implementarlas si se lleva a cabo otras investigaciones, ya que lograría un entendimiento aun mayor de la variable. 


\section{Declaración de financiamiento y de conflictos de interés:}

El estudio fue financiado por los autores, quienes declaran no tener algún tipo de conflicto de interés en la investigación realizada.

\section{Correspondencia}

Brandon Apaza Ramirez

Correo electrónico:

brandonapaza@upeu.edu.pe

Maryuri Pérez Coaquira

Correo electrónico

maryuriperez@upeu.edu.pe

\section{REFERENCIAS BIBLIOGRÁFICAS}

Aziz, S., Adkins, C., Walker, A., \& Wuensch, K. (2010) Workaholism and work-life imbalance: does cultural origin influence the relationship? International Journal of Psychology, 45(1), 72-79. Recuperado de: https://www.researchgate.net/ publication/51761635_Workaholism_and_worklife_imbalance_Does_cultural_origin_influence_ the_relationship

Colín, C. y Simón, N. (2014) Adicción al trabajo, satisfacción y desempeño laboral en ejecutivos mexicanos. Psicología Iberoamericana, 22(2), 16-24. Recuperado de: http://www.redalyc.org/ articulo.oa?id=133938134003

Del Líbano, M., Llorens, S., Schaufeli, W. y Salanova, M. (2006). Adicción al trabajo: conceptos y evaluación. Riesgos Laborales, 27, 23-30.

Doerfler, M. C. y Kramer, P. P. (1986). Workaholism: sex and sex role stereotyping among female professionals. Sex Roles, 14, 551-560.

Fassel, D. (1990). Working ourselves to death: the high costs of workaholism, the rewards of recovery. San Francisco, California: Harper Collins.

Flowers, C. y Robinson, B. (2002) A Structural and Discriminant Analysis of the Work Addiction Risk Test. Educational and Psychology Measurement, 62(3). Recuperado de: http://journals.sagepub. com/doi/abs/10.1177/00164402062003008

INEGI (2012). Encuesta nacional de ocupación y empleo: conjunto de datos población ocupada. Recuperado de http://www.inegi.org.mx/lib/ olap/consulta/general ver4/MDXQueryDatos. asp?prog=enoe2011_po

Kanai, A., Wakabayashi, M. \& Fling, S. (1996). Workaholism among employees in Japanese corporations: an examination based on the Japanese version of workaholism scales. Japanese Psychological Research, 38(4), 192-203 https://onlinelibrary.wiley.com/doi/ abs/10.1111/j.1468-5884.1996.tb00024.x

Merino, E., Boada, J. y Prizmic, A. (2014) The Relationship between Irritation at Work and Workaholism in a Spanish Multi-Occupational Sample. University Psychology, 13 (2), 15-27. Recuperado de: http://revistas.javeriana.edu.co/ index.php/revPsycho/article/view/3510/7106

Mosier, S. K. (1983). Workaholics: an analysis of their stress of work commitment. Austin, Texas: University of Texas Press

Oates, W. (1971). Confessions of a workaholic: the facts about work addiction. Nueva York: World Publishing.

Schaufeli, W., Bakker, A., van der Heijden, F. y Prins, J. (2009)Workaholism, burnoutand well-beingamong junior doctors: The mediating role of role conflicto. Work y Stress, 23(2), 155-172. Recuperado de: https://pdfs.semanticscholar.org/7003/ d0d52460efa4a32fd202962386fd6248368b.pdf

Scott, K., Moore, K., Miceli, M. (1997) An Exploration of the Meaning and Consequences of Workaholism. Human Relations, 50(3), 287314. Recuperado de: https://link.springer.com/ article/10.1023/A:1016986307298

Sorensen, K. y Feldman, D. (2006) Dimensions, antecedents, and consequences of workaholism: a conceptual integration and extensión. Journal of Organizational Behavior, 28(1), 111-136. Recuperado de: https://onlinelibrary.wiley.com/ doi/full/10.1002/job.424

Spence, J. y Robbins, A. (1992) Workaholism: Definition, measurement, and preliminary results .

AmericanPsychological Association, 58(1), 160178. Recuperado de: http://psycnet.apa.org/ record/1992-18384-001

Recibido: 20/02/2020

Aceptado: 15/04/2020 